\title{
REC BEAM PIPE TEMPERATURE CONTROL
}

\author{
Z. Greenwald, S. Greenwald and D. H. Rice * \\ Laboratory of Nuclear Studies, Cornell University, Ithaca, NY 14853
}

\begin{abstract}
In CESR the clearance between the beam pipe and the REC (Rare Earth Cobalt) magnet is only $1 \mathrm{~mm}$. The $160 \mathrm{~cm}$ beam pipe in this section is sloped and has steps to mask the detector from radiation. During high energy operation the beam pipe temperature increases due to synchrotron radiation, higher order modes power loss and image current, and effects the temperature of the REC magnet via convective and radiative heat transfer. The temperature change is proportional to the beam current. This causes a current dependent change in the REC magnetic field strength, resulting in a betatron tune shift of about $\Delta Q=0.011 /{ }^{\circ} \mathrm{C}$ for each quadrupole. To prevent the temperature changes of the beam pipe in this section, a sensitive temperature control cooling system was developed. The temperature of the beam pipe is stabilized within $\pm 0.1^{\circ} \mathrm{C}$. The loss parameter of the sloped pipe and power dissipated by synchrotron radiation was calculated from temperature measurements and used to estimate performance at higher current.
\end{abstract}

\section{INTRODUCTION}

The REC Quadrupole $\mathrm{Sm}_{-} \mathrm{Co}_{5}$ material has a magnetization temperature dependence of $-0.04 \%{ }^{\circ} \mathrm{C}$ (see reference [1]). In order to avoid a shift in the vertical tune due to changes in the REC magnetic field the temperature of the inner side of the REC must be controlled to within $\pm 0.1^{\circ} \mathrm{C}$. The main source of heating the inner side of the REC is the beam pipe whose temperature changes with the beam. Since the outer radius of the beam pipe inside the REC is only $1 \mathrm{~mm}$ smaller then the inner radius of the REC, the beam pipe inside the REC must be kept at constant temperature with fluctuation smaller then $\pm 0.1^{\circ} \mathrm{C}$ during all times with or without beam in CESR.

\section{TEMPERATURE CONTROL}

A block diagram of the control system is shown in Figure 1. The coolant (PF 200) temperature is measured at the output of the beam pipe section (point 2 on Figure 1) by a thermistor with high resolution (see Appendix I) and maintained constant by changing the coolant temperature at the input (point 1 on Figure 1) when the beam pipe is heated by synchrotron radiation and higher order modes. Figure $2 b$ shows the profile of the input temperature needed to keep the pipe temperature constant for the beam current shown in Figure 2a. A constant shift of about $0.3^{\circ} \mathrm{C}$ exists between the input and the output thermistors due to electronic offset. The coolant temperature

\footnotetext{
* Work supported by the National Science Foundation
}

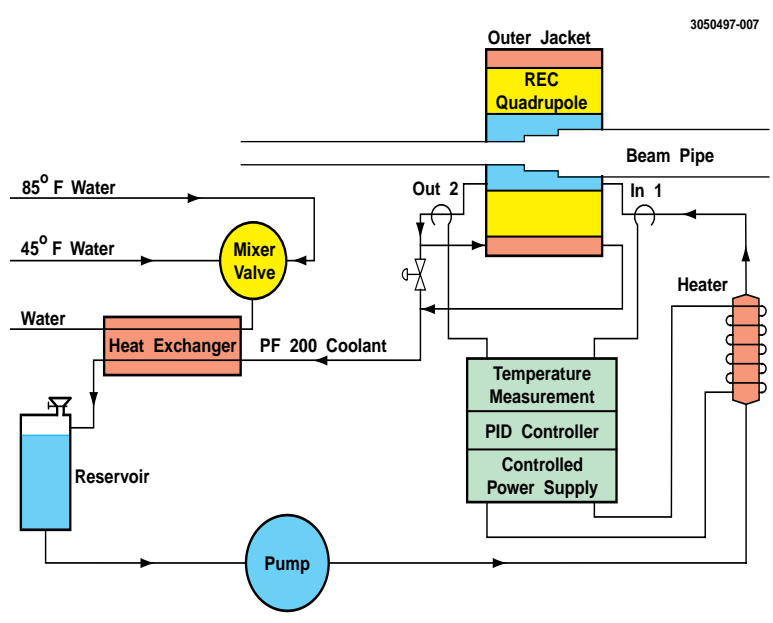

Figure 1: Block Diagram of the Control System

is regulated by a heater which also mixes the fluid making its temperature more uniform. The control system was designed to have a time constant of 3 minute in order to have a stable closed loop feedback and minimize the amount of walking. During injection when the filling rate is few ma per minutes the system is able to keep the beam pipe temperature very stable. In the case of a beam loss or total beam damp it takes the control system about 5 minutes to restabilize the beam pipe temperature, but even in this case the deviation in the beam temperature is only $0.1^{\circ} \mathrm{C}$ as can be seen from Figure $2 b$.

\subsection{The Heater}

The heater is built out of two concentric pipes 55 inches long, with a spiral divider in between. The coolant flows between the two pipes in a 0.1 inch gap along the spiral divider. Three heat tapes (1.5 KWatts each), connected to the regulating power supply, are wrapped on the outer pipe. The regulated voltage for the heat tapes that corresponds to the heating of the beam pipe due to the beam current shown in Figure $2 a$, is seen in Figure $2 c$. Note that the controlled voltage has to compensate not only for the heating due to the beam current but also the changes in the $45^{\circ} \mathrm{C}$ and $85^{\circ} \mathrm{C}$ water mix which cools down the coolant via a heat exchanger.

\subsection{The Controller}

The controller is regulating the power supply of the heater. It uses of an OMEGA PID[2] unit and a variable DC level shifter [3]. The DC shifter converts the desired REC beam 


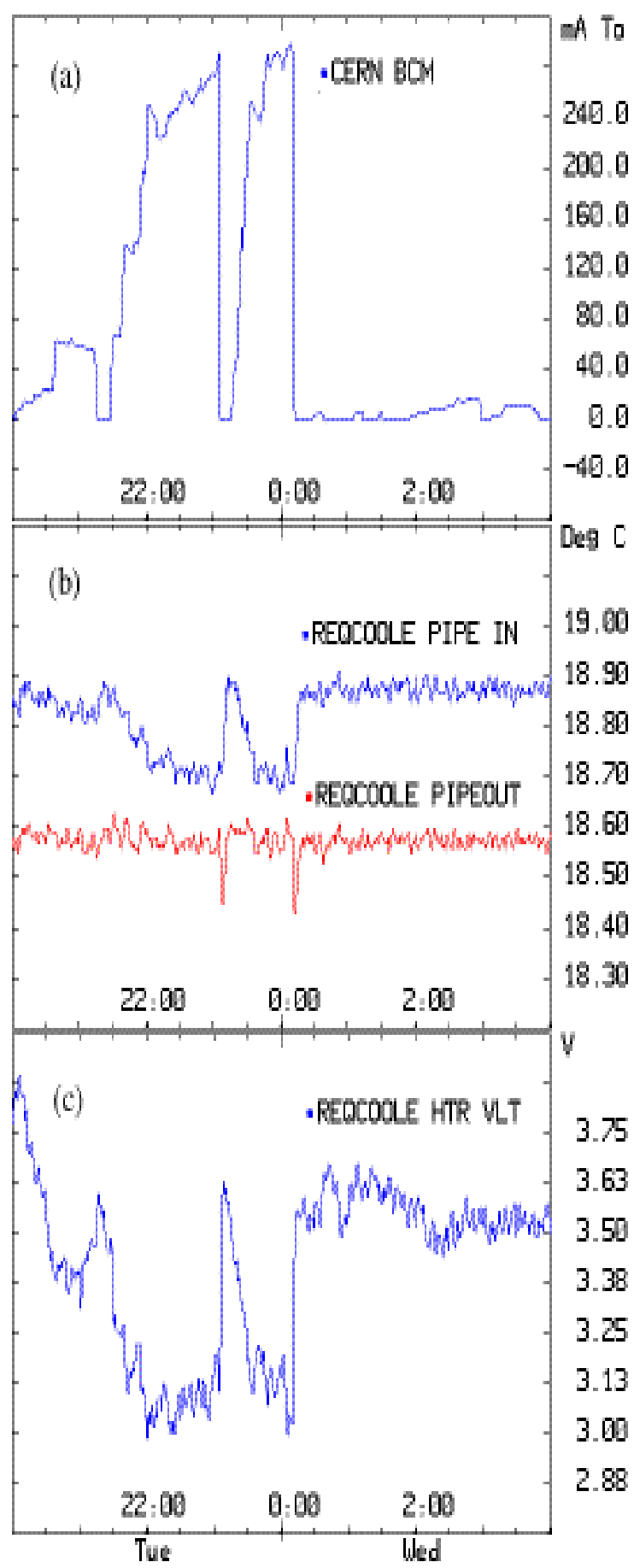

Figure 2: a. Positrons Current in the Storage Ring. b. Profile of the coolant input temperature needed to keep the pipe temperature constant, and the corresponding coolant output temperature. c. Regulated voltage corresponds to the heating due to the current in part a. Time scale is in hours.

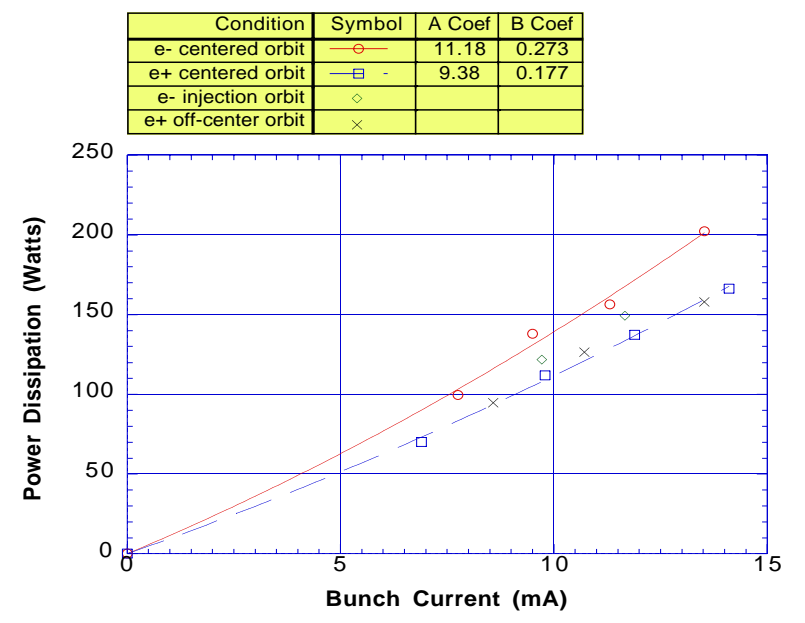

Figure 3: Power Dissipated in the WEST REC pipe by electrons and in the EAST REC pipe by positrons

pipe temperature to $2.5 \mathrm{Volts}$, which is the center of the PID $(0-5) V$ operating range. When the beam pipe is heated up by the beam, or the water temperature changes, the PID controller output can change up to $\pm 2.5 \mathrm{~V}$ corresponding to $\pm 10^{\circ} \mathrm{C}$.

\section{REC OUTER JACKET COOLING}

Some of the REC beam pipe stabilized coolant at the output side is diverted to the REC copper external jacket with brazed on tubes in order to stabilize the outer temperature of the REC magnets. This temperature is not as critical as the beam pipe temperature.

\section{MEASURED PRESENT POWER DISSIPATION}

As explained above, the temperature decrease in the input compensates the heating of the pipe, and thus the input temperature change is directly proportional to the power dissipated in the pipe. The power dissipation in the west REC pipe by electrons and in the east REC pipe by positrons, for several beam currents (18 bunches / beam), going through the center of the pipe is shown in Figure 3. The data were fitted to the function: $P=A \cdot I_{b}+B \cdot I_{b}^{2} ; A, B$ are constant. $I_{b}$, is the bunch current. The term $A \cdot I_{b}$ represents the dissipated power due to synchrotron radiation (linear with bunch current), and the term $B \cdot I_{b}^{2}$ represents the power loss due to higher order modes (proportional to square of the bunch current). At these currents the heating due to image currents is about 5 Watts [4]. The second set of data corresponds to the power dissipated in the pipe when the electron beam was displaced by a sine-like closed orbit distortion (generated by electrostatic separators to avoid parasitic beam-beam interactions). 


\begin{tabular}{|c|c|c|c|c|c|c|}
\hline \multirow{2}{*}{$\begin{array}{l}C E S R \\
\text { Upg- } \\
\text { rade }\end{array}$} & \multirow[b]{2}{*}{$\mathrm{Nb}$} & \multirow[b]{2}{*}{$\begin{array}{c}I b \\
(\mathrm{ma})\end{array}$} & \multicolumn{2}{|c|}{ WEST } & \multicolumn{2}{|c|}{ EAST } \\
\hline & & & $\begin{array}{c}P_{H O M} \\
\text { (W) }\end{array}$ & $\begin{array}{l}P_{S R} \\
(\mathrm{~W})\end{array}$ & $\begin{array}{c}P_{H O M} \\
\text { (W) }\end{array}$ & $\begin{array}{l}P_{S R} \\
(\mathrm{~W})\end{array}$ \\
\hline $\mathrm{Ph}$ II & 27 & 11.1 & 50.6 & 186.3 & 32.7 & 156.3 \\
\hline $\mathrm{Ph}$ III & 45 & 11.1 & 84.4 & 310.5 & 54.6 & 260.5 \\
\hline
\end{tabular}

Table 1: Estimated Power Dissipation in the REC Magnet for the Planed CESR Upgrades.

\section{CALCULATED FUTURE POWER DISSIPATION}

The above power dissipation was measured for 18 bunches. A general expression as function of the number of bunches $N_{b}$ is derived.

\subsection{Loss Parameter Calculation}

It is assumed that all the power measured is generated by heating in the REC itself. The Loss Parameter $k$ is calculated from $B$ extracted by the curve fitting to the data in Figure 3. $f_{R E V}$ is the revolution frequency, and $I_{B}$ bunch current.

$$
\begin{gathered}
P_{H O M}=k \cdot \frac{N_{b}}{f_{R E V}} \cdot I_{B}^{2}=B \cdot I_{B}^{2} \\
k=\frac{B \cdot f_{R E V}}{N_{b}}
\end{gathered}
$$$$
k_{W e s t}=5.94 \cdot 10^{-3} \mathrm{~V} / p C
$$$$
k_{\text {East }}=3.85 \cdot 10^{-3} \mathrm{~V} / \mathrm{pC}
$$$$
P_{\text {HOMWest }}=1.52 \cdot 10^{4} \cdot N_{b} \cdot I_{B}^{2} \text { watts }
$$$$
P_{\text {HOMEast }}=0.983 \cdot 10^{4} \cdot N_{b} \cdot I_{B}^{2} \text { watts }
$$

\subsection{Synchrotron Radiation Calculation}

The constant $A$ extracted from figure 3 was measured with 18 bunches. Thus the contribution from radiation is:

$$
\begin{gathered}
P_{S . R .}=\frac{A \cdot N_{b}}{18} \cdot I_{B} \\
P_{\text {S.R.West }}=621 . \cdot N_{b} \cdot I_{B} \text { watts } \\
P_{\text {S.R.East }}=521 \cdot \cdot N_{b} \cdot I_{B} \text { watts }
\end{gathered}
$$

\subsection{Future Estimation of Power Dissipation in REC pipe}

We plan to have a total of $300 \mathrm{ma} /$ beam carried by 9 trains with 3 bunches in a train in CESR phase II, and $500 \mathrm{ma} /$ beam carried by 9 trains with 5 bunches in a train in CESR phase III. The distribution of the calculated dissipated power in the WEST REC pipe due to the electron and in the EAST REC pipe due to positrons are summarized in Table 1.

\section{CONCLUSIONS}

After installing the temperature control system of the REC pipe we did not have any more fluctuations in the vertical tune due to temperature changes in the REC quadrupoles. The estimated total heating of each REC beam pipe will include also HOM heating by the other species. That means that the expected total power dissipation in Phase II will be 290 and 220 Watts for the WEST and EAST REC beam pipe respectively. In Phase III the expected total power dissipation will be 480 and 370 Watts for the WEST and EAST REC beam pipe respectively (Assuming we will be using similar optics in CESR). The main reason for the difference between the west and the east is due to different beam bumps of both sides of the interaction point that are put in to minimize the radiation into CLEO.

\section{APPENDIX}

\subsection{High Resolution Temperature Measurement}

The resolution of the measured temperature should be of the order of hundredth of a degree in order to stabilized the temperature with a $0.1^{\circ} \mathrm{C}$. A YSI 44005 precision thermistor was connected to $R=3 K \Omega$ resistor bridge, to balance the resistance of the thermistor at the center of the desired temperature range $\left(25^{\circ} \mathrm{C}\right.$ in this case). The bridge output voltage was connected to a difference amplifier. See reference [5]. The output voltage is:

$$
V_{o}=V_{b}\left(1+\frac{2 \cdot R_{2}}{R_{1}}\right)\left(\frac{R-R_{t h}}{R+R_{t h}}\right)
$$

where $V_{b}$ is a stabilized DC power supply, and $R_{1}, R_{2}$ are the amplifier high precision resistors.

for $R_{t h}=R+\Delta R_{t h}$ with $R>\Delta R_{t h}$ the output voltage

$$
V_{o}=V_{b}\left(1+\frac{2 \cdot R_{2}}{R_{1}}\right)\left(\frac{-\Delta R_{t h}}{2 R+\Delta R_{t h}}\right) \cong-G \Delta R_{t h}
$$

is linear with the temperature. The gain $G$ can be adjusted to give high resolution.

\section{REFERENCES}

[1] S. W. Herb, "Construction and Measurement of Large Permanent Magnet Quadrupoles",IEEE PAC 1987, catalog no. 87CH2387-9.

[2] CN76000 "Microprocessor - Based Temperature Controller" by OMEGA.

[3] D. Sagan, "REQ Beam Pipe Temperature Stabilization",CON 94-15 June, 1194.

[4] Z. Greenwald, "REC Pipe Temperature Control" drawing no. 6058-001 (05-1995).

[5] Z. Greenwald, "REC Temperature Readout" drawing no. 6056-021 (06-1995). 